Apidologie, 1980, 11 (1), 3-15.

\title{
GENETISCH BEDINGTE UNTERSCHIEDE IM LERNVERHALTEN DER HONIGBIENE (APIS MELLIFICA CARNICA)
}

\section{Différences d'origine génétique dans le comportement d'apprentissage de l'abeille (Apis mellifica carnica)}

\author{
Ingeburg OTT und Dorothea BRÜCKNER \\ Zoologisches Institut der Universität \\ Seidlstr. 25, 8 München 2
}

\begin{abstract}
ZUSAMMENFASSUNG
Um die Frage zu klären, ob das Lernverhalten der Kärntner Honigbiene durch Reduzierung der genetischen Variabilität verändert werden kann, wurden Farblernversuche mit Arbeiterinnen aus ingezüchteten und nichtingezüchteten Völkern durchgeführt. Die Ergebnisse zeigen, dass Arbeiterinnen aus ingezüchteten Völkern die Dressurmarke langsamer lernen und ein niedrigeres Niveau in ihren Lernkurven (\% Richtigwahlen) erreichen als Arbeiterinnen aus nicht ingezüchteten Völkern. Die möglichen Ursachen für diese Unterschiede werden diskutiert.
\end{abstract}

\section{SUMMARY \\ GENETIC DIFFERENCES IN THE LEARNING BEHAVIOUR OF THE HONEYBEE (APIS MELLIFICA CARNICA)}

In order to find out if the learning behaviour of honeybees (Apis mellifera carnica) is modified by a reduction in genetic variability, workers from inbred and non-inbred colonies were trained in color choice tests and their learning performance compared. The results show, that workers from inbred colonies ( 3 unrelated lines) learned the color mark slower and reached lower levels in their learning curves (\% correct choices) than workers from non-inbred colonies. The possible causes of this difference are discussed.

\section{EINLEITUNG}

Die folgende Untersuchung wurde durchgeführt, um $\mathrm{zu}$ klären, ob das Lernverhalten bzw. die Lernleistung von Arbeiterinnen der Kärntner Honigbiene (A pis mellifica carnica) durch Reduzierung der genetischen Variabilität verändert wird. 
In mehreren Untersuchungen zum Lernvermögen von Arbeiterinnen verschiedener Rassen der Honigbiene (Apis mellifica) konnte gezeigt werden, dass rassenspezifische Unterschiede im Lernverhalten bestehen (LINDAUER, 1970; MENZEL, FreudEL und RüHL, 1973; Hoefer und LiNdauer, 1975, 1976). Daher lag die Vermutung nahe, dass eine Reduzierung der genetischen Variabilität zu einer Veränderung des Lernverhaltens innerhalb einer Rasse führen könnte. Das an Arbeiterinnen der Kärtner Rasse zu untersuchen, war Aufgabe der vorliegenden Arbeit.

Untersuchungen an verschiedenen Merkmalen von Arbeiterinnen (z. B. Flügelsymmetrie, Temperaturregulierung und Rekrutierungsvermögen) haben gezeigt, dass das haplodiploide System der Honigbiene durch Reduzierung der genetischen Variabilität erheblich beeinflusst wird (BrüCKNER, 1975, 1976, 1978). Die Annahme, dass sich Inzucht in haplo-diploiden Systemen nicht so einschneidend auswirkt wie in diploiden Systemen trifft nach diesen Untersuchungen nicht zu. Das Lernverhalten der Arbeiterinnen sollte auch unter diesem Gesichtspunkt weitere Ergebnisse liefern.

\section{MATERIAL}

Die Versuche wurden mit Arbeiterinnen aus ingezüchteten und nicht-ingezüchteten Völkern der Kärntner Honigbiene (A.m.c.) durchgeführt. Insgesamt wurden 6 Inzucht- und 6 Nicht-Inzuchtvölker getestet. Die Inzucht wurde mit Hilfe von künstlicher Besamung der Bienenkönigin erreicht.

Die ingezüchteten Völker entstammten 3 nicht verwandten Linien (I1, I6, I19, je 2 Völker von jeder Linie) mit verschiedenen Inzuchtsystemen. Die getesteten Arbeiterinnen stellten die 4. (I19) bzw. 5. (I1 und I6) Inzuchtgeneration dar. Die berechneten Inzuchtkoeffizienten $(F)$ waren wie folgt : I1 : F = 59,4\%, I6: $\mathrm{F}=64 \%, \mathrm{I} 19: \mathrm{F}=46,9 \%$. In der Auswertung werden die Ergebnisse der Arbeiterinnen aller Inzuchtlinien in manchen Berechnungen zusammengefasst, da die Inzuchtkoeffizienten hinreichend nahe beieinander liegen.

Ein Inzuchtskoeffizient von $100 \%$ kann bei Arbeiterinnen der Honigbiene nicht erreicht werden, da multiple Allelie am Genort, der das Geschlecht bestimmt, vorliegt : Weibchen entstehen nur, wenn der Genort heterozygot ist (WOYKe, 1965). Die getesteten NI-Bienen ( $F=O)$ stammten aus 6 Völkern, die , untereinander und mit den Inzuchtiinien nicht verwandt waren; eine Ausnahme davon macht ein Volk, das einer Kreuzung von 2 inzuchtlinien $(I 1 \times 119)$ entstammte.

Alle Völker waren in Drei-Wabenkästen untergebracht und befanden sich im selben Bienenstand im Garten des Zoologischen Instituts. Es wurde darauf geachtet, dass die Volksgrössen vergleichbar waren. Die Versuche wurden in Sommer 1978, Mai-September durchgeführt. Inzucht- und Nicht-Inzuchtvölker wurden in abwechselnder Reihenfolge getestet.

\section{METHODE}

Die Versuchsmethode entsprach der von MEnzel, Freudel und RüHL (1973) verwendeten (horizontale Anordnung) : Jeder Lernversuch wurde mit einer einzigen Biene durchgeführt; es wurden je 
10 Bienen pro Volk getestet. Die Versuchsbiene $(\mathrm{Vb})$ wurde $3 \mathrm{x}$ auf einem schwarzen Kreuz (Schwarzkreuz) in der Mitte eines runden grauen Tisches belohnt (=Vordressur). Auf die 3. Belohnnung folgte ein 4 minütiger Test, in dessen Verlauf die $\mathrm{Vb}$ zwischen Gelb- und Blaukreuz wählen musste (siehe Abb. 1). Dieser Test wird als Spontanwahl bezeichnet, da die Vb vorher weder auf Gelb- noch auf

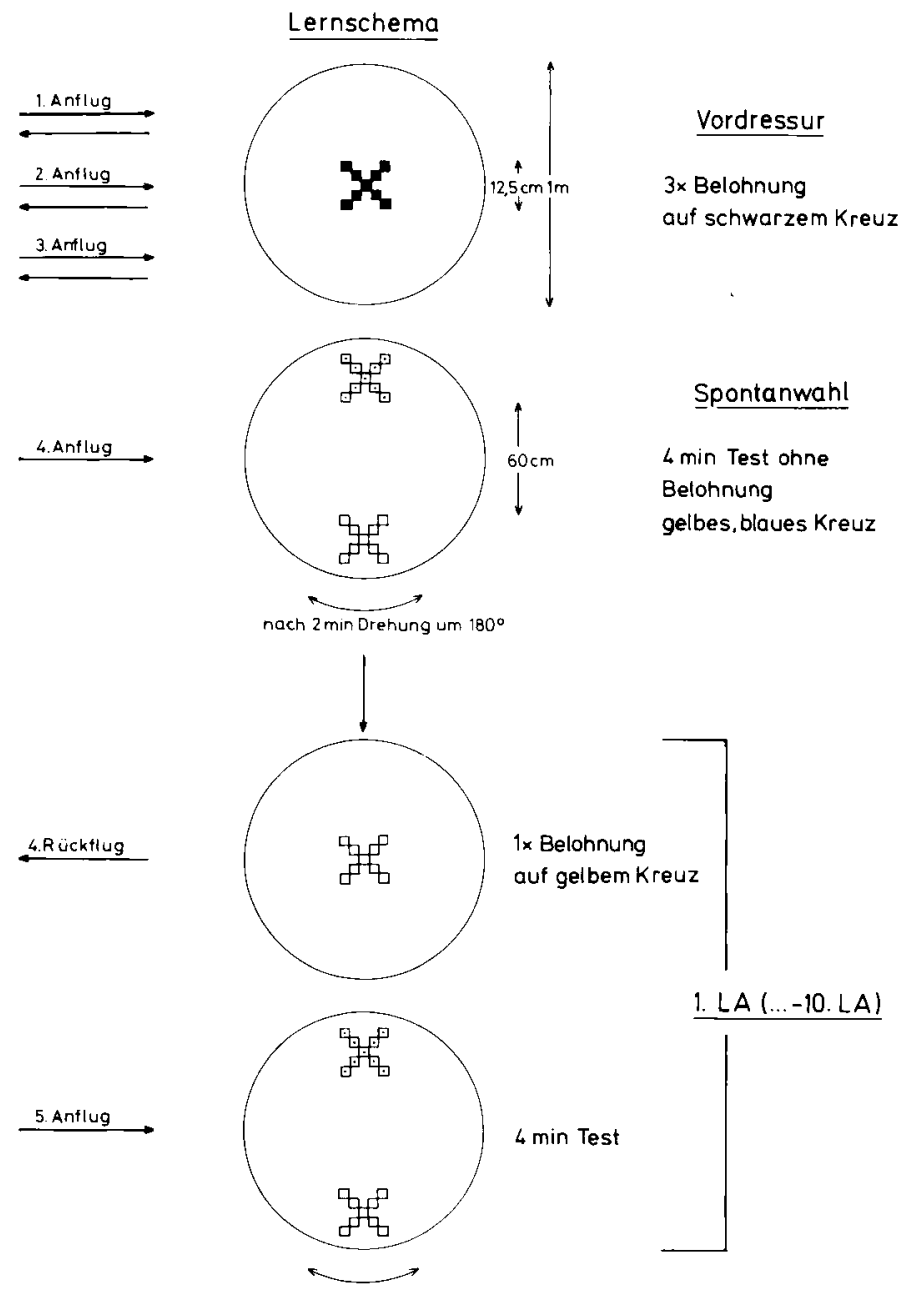

Aвв. 1. - Belohnungs- und Testschema. Erklärung siehe Text.

FIG. 1. - Schéma de la récompense et du test. Voir explications dans le texte.

Blaukreuz belohnt worden war. Nach der Spontanwahl wurde die $\mathrm{Vb}$ zum 1. Mal auf Gelbkreuz belohnt: daran schloss sich wieder ein 4 minütiger Test an. Während der Tests wurde das Suchverhalten der Vb protokolliert. Die Einheit Belohnung und Test wird als Lernakt (LA) bezeichnet. 
Insgesamt wurde jede $\mathrm{Vb} 10 \mathrm{x}$ belohnt $(10 \mathrm{LA})$. Die Versuchsanordnung befand sich in einem Glashaus; die Entfernung zwischen Tisch und Bienenstand betrug ca. $80 \mathrm{~m}$. Die statistische Auswertung erfolgte einerseits nach dem $\mathbf{t}$-Test, wobei alle Unterschiede als signifikant bezeichnet wurden, bei denen der Test weniger als $5 \%$ Irrtums-wahrscheinlichkeit ergab; andererseits mit Hilfe des Zeichentests von v.d. WAERDEN und Nievergelt (1956) für die mittleren Lernkurven der Inzucht- und Nicht-Inzuchtbienen. Bei der zweiseitigen Anwendung dieses Tests wird festgestellt, mit welcher Irrtumswahrscheinlichkeit die Werte einer Kurve durchschnittlich grösser oder kleiner sind als die Werte einer zweiten Kurve. Für $p<0,01$ gilt der Unterschied als stark gesichert, für $p<0,05$ als schwach gesichert.

\section{ERGEBNISSE}

Das Suchverhalten einer $\mathrm{Vb}$ im Test wurde nach den 3 Kategorien Anflug (A), Antippen (T) und Setzen (S) auf die Marken protokolliert. Daraus wurden für jede Biene die \% Richtigwahlen in den Tests nach jeder Belohnung (bis zur 10. Belohnung) berechnet. Die Anflugs- Entscheidungen (A \%) wurden gesondert von den Tipp- und Sitz-Entscheidungen ( $T+S \%$ ) ausgewertet. Man erhält insgesamt weniger $T+S$ Entscheidungen und ihre Auswertung ergibt steilere Lernkurven, da Antippen und Sitzen präziser gesteuert werden als Anfliegen.

Abb. 2 zeigt die Auswertung der Anflugs-Entscheidungen. Die Bienen der 6 NIVölker $(60 \mathrm{Vb})$ wurden bei der Auswertung zu einer Gruppe zusammengefasst und den 3 Inzuchtlinien (je $20 \mathrm{Vb}$ ) gegenübergestellt.

In der Spontanwahl zeigen die NI-Bienen eine niedrigere Wahltendenz gegenüber dem Gelbkreuz (in \%) als die Inzuchtbienen.

Um die Lernkurven in ihrem tatsächlichen Verlauf besser vergleichen zu können, wurden sie in Abb. 3 auf ihren jeweiligen Ausgangswert bezogen und der prozentuale Lernzuwachs in Abhängigkeit von der Zahl der Belohnungen angegeben. Die Lernkurve der NI-Bienen verläuft steiler und erreicht ein höheres Niveau als die Lernkurven der Inzucht-Bienen. Die Lernkurven der 3 I-Linien zeigen etwa den gleichen Verlauf. Die Bienen aus diesen Völkern lernen das Gelbkreuz langsamer und erreichen ein deutlich niedrigeres Lernniveau als die NI-Bienen. In der statistischen Auswertung mit dem tTest sind alle Werte der Lernkurven (Abb. 3) der NI-Bienen gegenüber denen der Inzuchtlinien mit $p<0,02$ signifikant unterschiedlich. Im Zeichentest ergibt sich ebenfalls mit $p<0,02$ ein gesicherter Unterschied.

Die Kurve der NI-Bienen weist nach der 5. Belohnung einen Knick auf; eine mögliche Erklärung für dieses Phänomen soll in der Diskussion gegeben werden. Die Auswertung der $\mathrm{T}+\mathrm{S}$-Entscheidungen (Abb.4) veranschaulicht deren präzisere Steuerung. Die T + S-Entscheidungen erreichen in beiden Gruppen ein höheres Niveau als die jeweilige Anflug-Lernkurve (auch diese Kurven wurden zum besseren Vergleich auf 0 als Ausgangswert umgerechnet).

Der Unterschied zwischen den Kurven der Inzuchtlinien und denen der NichtInzuchtbienen ist nicht so gross wie bei der A-Auswertung, jedoch liegen die Kurven der Inzuchtlinien in allen Punkten unterhalb der Kurven der NI-Bienen. 


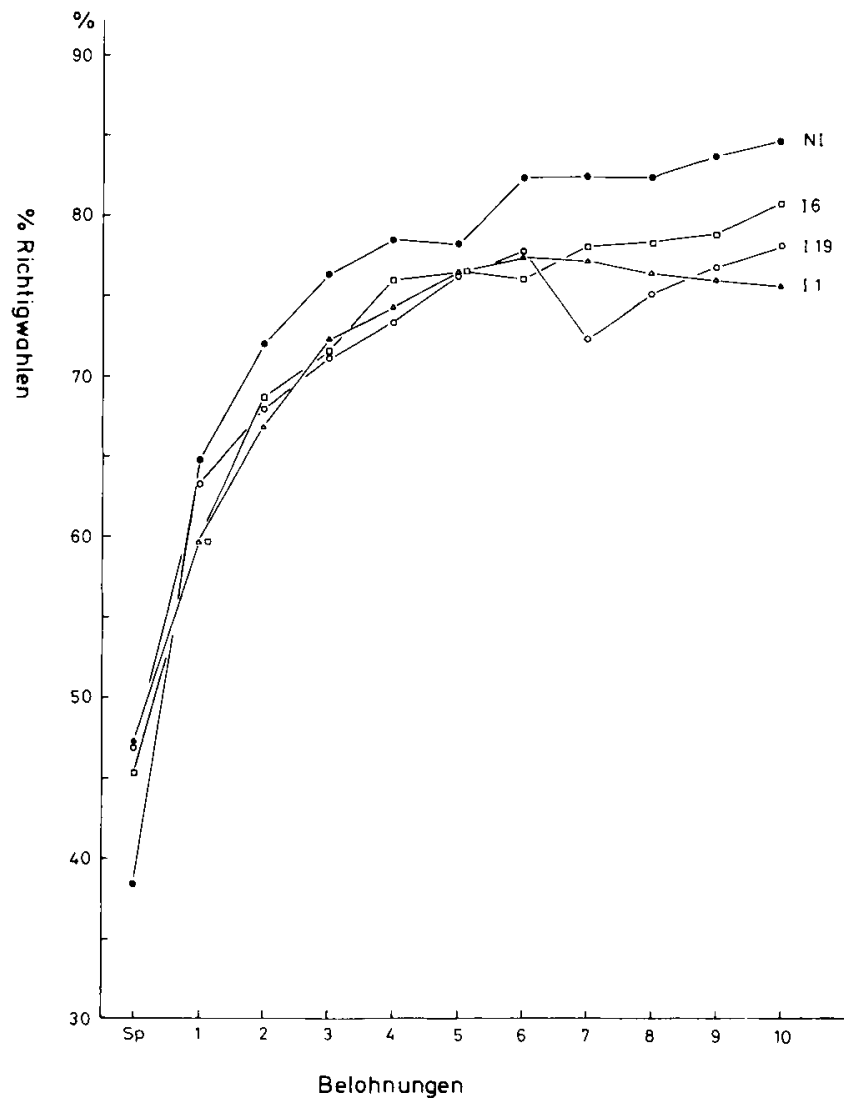

Aвв. 2. - Mittlere Lernkurven der Nicht-Inzuchtbienen (NI, 60 Versuchsbienen, Vb) und der Inzuchtbienen I 1, I 6 und I 19, je $20 \mathrm{Vb}$ ) bei der A uswertung der Anflugs-Entscheidungen.

Abszisse : Anzahl der Belohnungen auf Gelbkreuz,

$\mathrm{Sp}=$ Spontanwahl

Ordinate : \% Richtigwahlen für Gelbkreuz Die Standardfehler der Mittelwerte wurden der Übersichtlichkeit halber nicht angegeben, sie liegen im Bereich von 0,6 bis $2,7 \%$ Richtigwahlen.

FIG. 2. - Courbes moyennes d'apprentissage des abeilles non-consanguines (NI, 60 ourrières testées: Vb et des abeilles consanguines $(11: 16 \mathrm{Vb}$ et $119: 20 \mathrm{Vb})$

d'après les choix de la catégorie $A$ "arrive en volant".

En abscisses: Nombre de récompenses sur la croix jaune $\mathrm{Sp}=$ choix spontané

En ordonnées : \% de choix justes pour la croix jaune. Les écarts-standard des moyennes n'ont pas été donnés pour des raisons de clarté, elles se situent aux alentours de 0,6 a $2,7 \%$ des choix justes.

Nach dem t-Test sind die Werte der Inzuchtlinien bis auf folgende Ausnahmen signifikant von denen der NI-Bienen verschieden : 1. LA, I19: NI; 4. LA, I6: NI; 5 . LA, alle Linien : NI; 8. LA, I6: NI und 10. LAI6: NI; bei der 5 . Belohnung tritt wie bei den A-Entscheidungen ein Knick in der Lernkurve der NI-Bienen auf. 


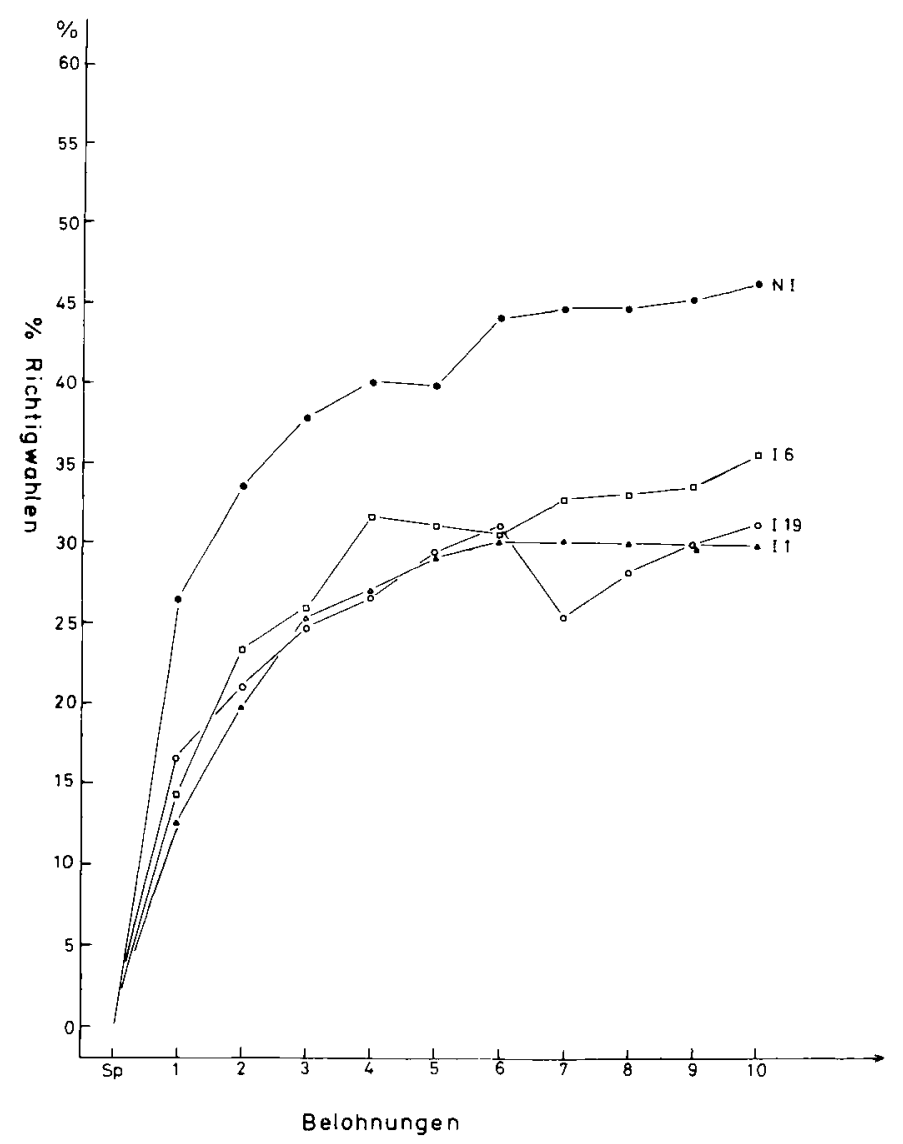

ABв. 3. - Mittlere Lernkurven der Nicht-Inzuchtbienen (NI, $60 \mathrm{Vb})$ und der Inzuchtbienen (I 1. I 6 und I 19, je 20 Vb) bei Auswertung der Anflugs-Entscheidungen.

Die Lernkurven sind auf 0 als Ausgangswert umgerechnet.

Abszisse : Anzahl der Belohnungen auf Gelbkreuz,

$\mathrm{Sp}=$ Spontanwahl

Ordinate : \% Richtigwahlen für Gelbkreuz. Die Standardfehler der Mittelwerte wurden der Übersichtlichkeit halber nicht angegeben, sie liegen im Bereich von 1,1 bis $3,1 \%$ Richtigwahlen.

FIG. 3. - Courbes moyennes d'apprentissage des abeilles non-consanguines (NI, $60 \mathrm{Vb})$ et des abeilles consanguines ( $11: 16 \mathrm{Vb}$ et $119: 20 \mathrm{BV}$ )

d'après les choix de la catégorie $A$ "arrive en volant". Les courbes dapprentissage sort ramenées au 0. En abscisses : nombre de récompenses sur la croix jaune

$$
\mathrm{Sp}=\text { choix spontané }
$$

En ordonnées : \% de choix justes pour la croix jaune. Les écarts-standard des moyennes n'ont pas été donnés pour des raisons de clarté; ils se situent aux alentours de 1,1 à $3.1 \%$ des chorx justes.

Da die Auswertung der A-Entscheidungen einen grösseren Lernunterschied als die Auswertung der T + S-Entscheidungen erbrachte wurde die Kopplung zwischen A- und $\mathrm{T}+\mathrm{S}$-Entscheidungen untersucht. Um festzustellen, ob eine unterschiedliche Kopplung 


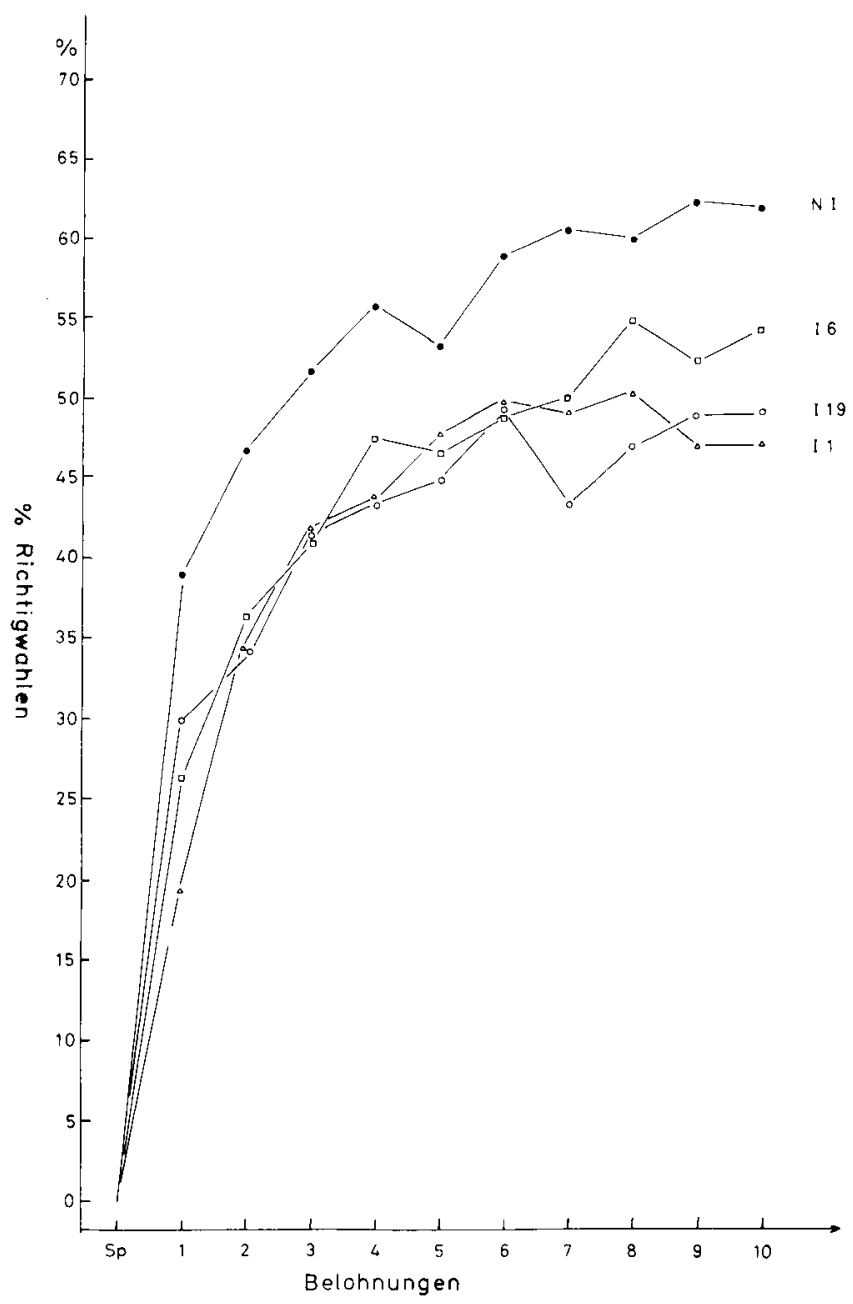

ABB. 4. - Mittlere Lernkurven der Nicht-Inzuchtbienen (NI, $60 \mathrm{Vb})$ und der Inzuchtlinien

I1, I6 und I19, je $20 \mathrm{Vb}$ ) bei Auswertung der Tipp- und Sitz-Entscheidungen.

Die Lernkurven sind auf 0 als Ausgangswert umgerechnet.

Abszisse : Anzahl der Belohnungen auf Gelbkreuz,

$\mathrm{Sp}=$ Spontanwahl

Ordinate : \% Richtigwahlen für Gelbkreuz. Die Standardfehler der Mittelwerte wurden der Übersichtlichkeit halber nicht angegeben, sie liegen im Bereich von 2,5 bis $6,1 \%$ Richtigwahlen.

FIG. 4. - Courbes moyennes d'apprentissage des abeilles non-consanguines (NI, $60 \mathrm{Vb}$ ) et des abeilles consanguines $(11: 16 \mathrm{Vb}$ et $119:$ chacune $20 \mathrm{Vb})$

d'après les choix de la catégorie $T+S$ "tapote " et "est posée ". Les courbes d'apprentissage ont été ramenées au 0 .

En abscisses : Nombre de récompenses sur la croix jaune $\mathrm{Sp}=$ choix spontané

En ordonnées : \% de choix justes pour la croix jaune. Les écarts-standard des moyennes n'ont pas été donnés pour des raisons de clarté; ils se situent aux alentours de 2,5 à $6,1 \%$ des choix justes. 
zwischen A- und T + S-Wahltendenz vorliegt, wurde für jede Gruppe (NI-Bienen, $n=60 /$ - Bienen, $n=60)$ der Quotient $\frac{A \%}{T+S \%} z u$ jedem Lernakt berechnet und in Abb. 5 als Kurve aufgetragen.

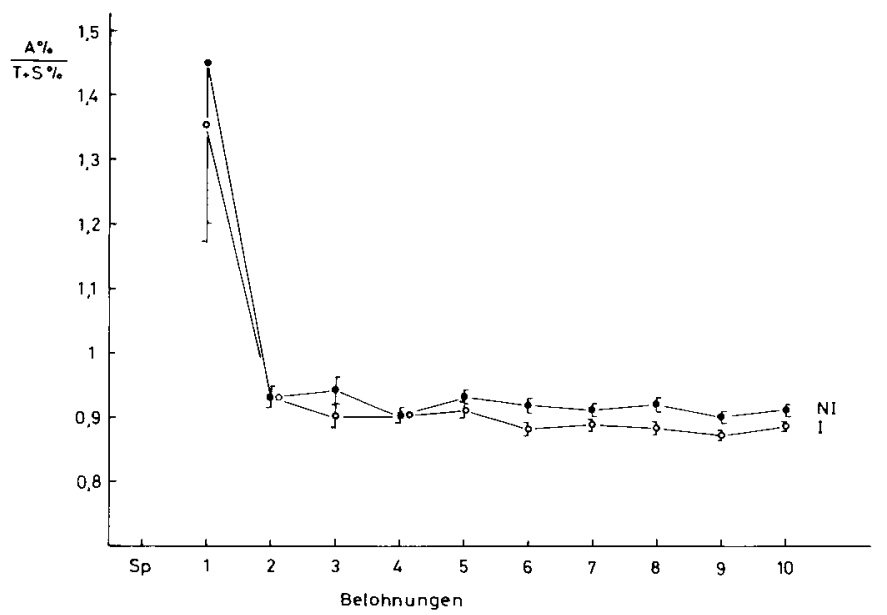

Aвв. 5. - Kopplung zwischen Anflug (A) - Wahltendenz und Tipp- und Sitz (T+S)-Wahltendenz im Lernverlauf (1.-10. Belohnung) bei Inzucht (1)-und Nicht-Inzucht-Bienen (NI).

Ordinate : Quotient aus der prozentualen Richtigwahl bei A- und T + S-Entscheidungen $\frac{\mathrm{A} \%}{\mathrm{~T}+\mathrm{S} \%}$. Mittelwerte und Standardfehler der Mittelwerte sind gegeben.

FIG. 5. - Corrélation entre la tendance de choix $A$ et la tendance de choix $T+S$ au cours de l'apprentissage

( $1^{e r}-10^{e}$ récompense) chez les ouvrières consanguines (l) et non-consanguines (NI). En abscisses : Nombre de récompenses

En ordonnées: Rapport du $\%$ de choix $A$ justes au $\%$ de choix $T+S$ justes $\frac{A \%}{T+S \%}$

In der Spontanwahl ergibt sich in allen 3 Verhaltenskategorien ein etwa gleicher. Prozentsatz der Wahltendenz (NI : 4,85/I : 4,83; in der Abb. nicht aufgeführt). Da daran anschliessend die Lernkurven in jedem Fall bei den $\mathrm{T}+\mathrm{S}$-Entscheidungen steiler ansteigen als bei den A-Entscheidungen folgt ein starker Abfall des genannten Quotienten.

Die Kurven der Nicht-Inzucht- und Inzuchtbienen liegen zwar sehr nahe beieinander, dennoch kann man $a b$ der 6 . Belohnung im t-Test alle Kurvenpunkte gegeneinander absichern $(p<0,02)$.

Im Zeichentest ist der Unterschied zwischen den Kurven mit $p<0,01$ ebenfalls stark gesichert. 
Die Inzuchtbienen weisen einen niedrigeren Quotienten $\frac{A \%}{T+S \%}$ auf, d.h. der Unterschied zwischen A- und T + S-Wahltendenz ist bei ihnen grösser als bei den NIBienen. Auf die mögliche Ursache für den Unterschied in der Kopplung zwischen Aund $\mathrm{T}+\mathrm{S}$-Wahltendenz ab der 6 . Belohnung soll in der Diskussion eingegangen werden.

Wie schon weiter vorn erwähnt, wird Tippen und Sitzen auf der Marke präziser gesteuert als Anfliegen allein. Daraus resultiert, dass Anflüge, auf die Tippen oder Sitzen folgt, sicherer sind als Anflüge ohne diese stärkere Entscheidung für die Marke. Treten Tipp- und Sitz-Entscheidungen im Verhältnis zu den A- Entscheidungen häufiger auf, so ergibt sich allein aufgrund dieser Verhältnisänderung eine bessere Wahl der Lernmarke auch im Anflug. Relativ mehr T + S-Entscheidungen haben eine steilere ALernkurve zur Folge.

Daher stellte sich die Frage, ob die bessere Wahl der Lernmarke im Anflug bei den NI-Bienen im Vergleich zu den I-Bienen auf ein günstigeres Verhältnis von A- zu $\mathrm{T}+\mathrm{S}$-Entscheidungen $\left(\frac{\mathrm{A}}{\mathrm{T}+\mathrm{S}}=\right.$ Suchstrategie$)$ zurückzuführen ist. Die Suchstrategie für beide Gruppen ist in Abb. 6 wiedergegeben.

Im ersten Bereich der Kurven zeigt sich ein Abfall des Quotienten (stärkere Zunahme der sicheren $\mathrm{T}+\mathrm{S}$-Entscheidungen), im zweiten Bereich pendeln sich die Kurven auf ein Niveau ein. Bis zur 4. Belohnung verlaufen die Kurven sehr ähnlich. Dann divergieren sie : Die I-Bienen verschlechtern ihre Suchstrategie geringfügig (weniger T + S-Entscheidungen, der Quotient nimmt zu), während die NI-Bienen ihre Suchstrategie geringfügig verbessern (mehr $\mathrm{T}+\mathrm{S}$-Entscheidungen, der Quotient nimmt $\mathrm{ab}$ ).

Testet man die Mittelwerte, die aus den Werten von der 1.-10. Belohnung für I und NI-Bienen berechnet sind, im t-Test gegeneinander, so ergibt sich ein signifikanter Unterschied (NI $\bar{x}=1,6, \mathrm{I} \bar{x}=1,7, p<0,001$ ). Bei Anwendung des Zeichentests ergibt sich ebenfalls ein mit $p<0,01$ stark gesicherter Unterschied.

\section{DISKUSSION}

Die für Inzucht- und Nicht-Inzuchtbienen gefundenen Lernunterschiede könnten durch genetische Unterschiede sowie durch Umweltfaktoren verursacht sein. Von MENZEL (1967) konnte jedoch gezeigt werden, dass Änderungen verschiedener äusserer Faktoren wie Lichtverhältnisse, Nektarangebot, Umgebungstemperatur und Testdauer keinen Einfluss auf den Lernverlauf hatten. $\mathrm{Da}$ in unseren Versuchen die Bedingungen am Dressurort für alle Versuchsbienen gleich gehalten wurden und nur Arbeiterinnen aus Völkern vergleichbarer Grösse verwendet wurden, nehmen wir an, dass die 


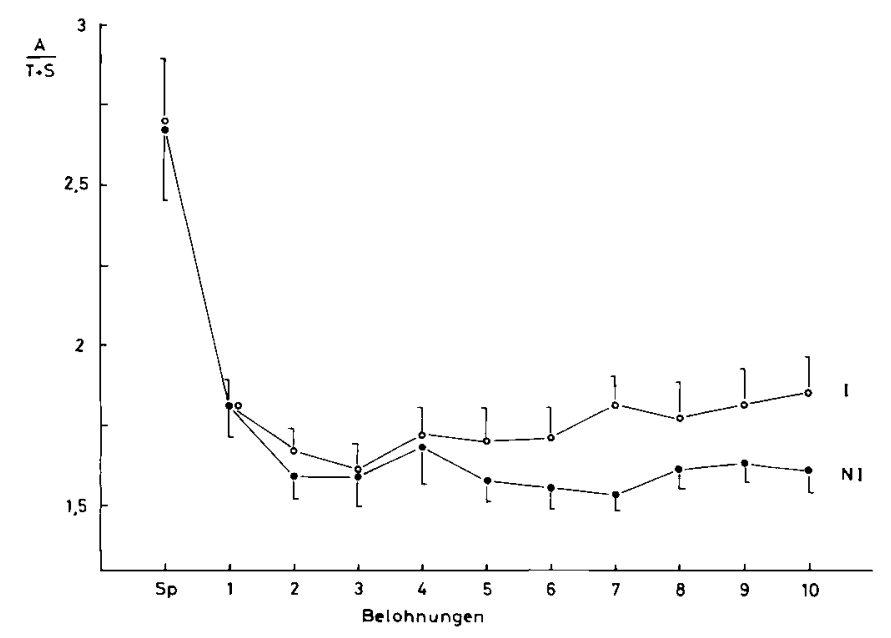

Авв. 6. - Suchstrategie $\frac{A}{T+S}$ im Lernverlauf (Sp - 10. Belohnung) für Inzucht (I)- und Nicht-InzuchtBienen (NI).

Abszisse : Zahl der Belohnungen, $\mathrm{Sp}=$ Spontanwahl

Ordinate : Quotient aus der Anzahl der Anflüge und der Tipp und Sitz-Entscheidungen im Test $\frac{\mathrm{A}}{\mathrm{T}+\mathrm{S}}$ Mittelwerte und Standardfehler der Mittelwerte sind gegeben.

FIG. 6. - Stratégie de recherche $\frac{A}{T+S}$ au cours de l'apprentissage (choix spontané $S p-10^{e}$ récompense)

pour des abeilles consanguines (I) et des abeilles non-consanguines (NI).

En abscisses: Nombre de récompenses

$\mathrm{Sp}=$ choix spontané

En ordonnées : Rapport du nombre de choix " arrive en volant " au nombre de choix " tapote " et " est posé " dans le test $\frac{\mathrm{A}}{\mathrm{T}+\mathrm{S}}$. Les moyennes et les écarts-standard sont donnés.

Unterschiede in den Lernkurven hauptsächlich auf den Unterschieden in der genetischen Konstitution der getesteten Arbeiterinnen beruhen.

Die schlechte Lernleistung der Inzuchtbienen könnte auf einer Schwächung der physiologischen Mechanismen der Gedächtnisbildung beruhen. Eine geringere Leistungsfähigkeit im physiologischen Bereich bei Inzuchtbienen wurde z.B. bei der Stoffwechselwärmeerzeugung gefunden (BRÜCKNER, 1975).

Nach Untersuchungen von Menzel, Erber und Masuhr (1974) und Erber (1972, 1975) geht die Einspeicherung einer Information über ein Kurzzeit- in ein Langzeitgedächtnis. Das Kurzzeitgedächtnis beruht auf neuronalen Mechanismen und kann durch verschiedene Behandlungsmethoden beeinflusst werden (z.B. Elektroschocks). Von der 3.-7. min. nach der Assoziation der Dressurmarke mit der 
Belohnung wird die Information ins Langzeitgedächtnis eingespeichert (Proteinsynthese), die getesteten Störungen zeigen immer weniger Auswirkungen, das LZG ist ihnen gegenüber unempfindlich. Die im LZG gespeicherte Information wird relativ lange behalten. Die in der vorliegenden Untersuchung gemessene Lernleistung stellt demnach einen Ausdruck des LZG dar. Die gefundenen Lernunterschiede könnten auf den verschiedensten physiologischen Veränderungen im LangzeitLernsystem beruhen.

Wie bei den Ergebnissen erwähnt, tritt ein " Knick » (plötzliche Verminderung der Richtigwahlen) in der Lernkurve der NI-Bienen beim 5. LA auf (bei 5 der 6 NI-Völker und 1 I-Volk). Dieses Phänomen wurde von Hoefer und Lindauer (1975) entdeckt; bei ihnen erfolgt der Knick beim 6. LA, da sie keine Vordressur durchführten). Auf Grund von Umlernversuchen, in denen dem 6. LA als Grenzbereich eine besondere lernphysiologische Bedeutung zukam, stellten HOEFER und LiNDAUER die Hypothese von 2 Lerneinheiten im Lernsystem (des Langzeitgedächtnisses) der Honigbiene auf, wobei der 6. LA das "Einklicken" der 2. Lernphase charakterisieren soll. Bei der vorliegenden Untersuchung tritt der Knick in der Lernkurve mit einer Ausnahme bei allen NI-Völkern auf. Das Fehlen dieser Charakteristik der Lernkurve bei Inzuchtbienen könnte ein Hinweis auf eine Veränderung im LZG der I-Bienen sein.

Auch bei der Auswertung der Kopplung zwischen A und $\mathrm{T}+\mathrm{S}$-Wahltendenz $\frac{\mathrm{A} \%}{\mathrm{~T}+\mathrm{S} \%} \mathrm{Abb} .4$ ergab sich ab der 6. Belohnung ein Unterschied in den Kurven, wobei die I-Bienen eine ungünstigere Kopplung aufweisen. Die Auswertung der Suchstrategie (Abb. 5) ergab einen grösseren Unterschied zwischen den beiden Gruppen ab der 5. Belonnung, wobei wieder die-I-Bienen ihre Suchstrategie verschlechtern.

Diese Ergebnisse könnten als eine Veränderung in der 2. Lerneinheit gedeutet werden. Damit wären sie gleichzeitig ein weiterer Hinweis auf das tatsächliche Vorhandensein von 2 Lerneinheiten im Lernsystem der Honigbiene. Da die (bisher hypothetischen) Lerneinheiten wahrscheinlich unterschiedliche physiologische Prozesse darstellen, könnte eine Schwächung der 2. Lerneinheit auf einer Veränderung ihrer physiologischen Basis durch Inzucht beruhen.

\section{DANK}

Wir möchten uns bei Herrn Dr. V. Maul für die Bereitstellung der ingezüchteten Völker sehr herzlich bedanken und bei Herrn Prof. Dr. M. RenNer, der uns das Gartenhaus zur Verfügung stellte. 


\section{RÉSUMÉ}

Dans le travail présent on a comparé le comportement d'apprentissage des ouvrières de colonies consanguines et de colonies non-consanguines de l'abeille carnolienne (Apis mellifica carnica).

On a testé de la façon suivante 6 colonies consanguines et 6 non-consanguines à raison de 10 ouvrières par colonie : les ouvrières subissaient un dressage avec récompense sur une marque coloré (jaune); chaque récompense était suivie d'un test, au cours duquel on classait le comportement de choix des ouvrières entre les couleurs jaune et bleu en 3 catégories, selon qu'elles arrivaient en volant (A), qu'elles tapotaient ou qu'elles étaient posées $(\mathrm{T}+\mathrm{S})$. Le processus d'apprentissage est décrit à l'aide de courbes d'apprentissage qui sont calculées à partir du \% de choix corrects $A$ et de choix corrects $\mathrm{T}+\mathrm{S}$.

La capacité d'apprentissage des ouvrières non-consanguines est meilleure que celle des ouvrières consanguines, que ce soit pour les ahoix A ou les choix $\mathrm{T}+\mathrm{S}$. La corrélation entre A \% et $\mathrm{T}+\mathrm{S} \%$ et la stratégie de recherche $\left(\right.$ rapport $\left.\frac{A}{T+S}\right)$ montre qu'il existe une différence entre les abeilles consanguines et les abeilles non-consanguines.

On discute des raisons possibles de la variation de cette capacite d'apprentissage, en admettant comme cause l'affaiblissement, lié à la consanguinité, des mécanismes physiologiques de la formation de la mémoire dans la mémoire à long terme.

\section{LITERATURVERZEICHNIS}

BRỦCKNER D., 1975. - Die Abhängigkeit der Temperaturregulierung von der genetischen Variabilität der Honigbiene (A pis mellifica). Apidologie 6 (4), 361-380.

BRÚCKNER D., 1976. - The influence of genetic variability on wing symmetry in honeybees (Apis mellifera). Evolution, Vol. 30 (1), 100-108.

BRÜCKNER D., 1976. - Vergleichende Untersuchungen zur Temperaturpräferenz von ingezüchteten und nicht-ingezüchteten Arbeiterinnen der Honigbiene (Apis mellifica). Apidologie 7 (2), 139-149.

BRƯCKNER D., 1976. - Nachweis von Heterosis für das Merkmal Temperaturregulierung bei der Honigbiene (Apis mellifica). Apidologie 7 (3), 243-248.

BRUंCKNER D., 1978. - Why are there inbreeding effects in haplo-diploid systems? Erolution 32 (2), 456-458.

BRÜCKNER D. - Untersuchungen zum Rekrutierungsvermögen von Arbeiterinnen aus Inzuchtvölkern (Apis mellifica carnica) (in Vorbereitung).

BRÜCKNER D., 1980. - Hoarding behaviour and life span of inbred, non inbred and hybrid honeybees (Apis mellifica) J. apic. Res. 19 (1).

ERber J., 1975. - The dynamics of learning in the honey bee (Apis mellifica carnica). J. comp. Physiol. 99 (1) und (2), 231-242.

HoEfer I. LiNDAuer M., 1975. - Das Lernverhalten zweier Bienenrassen unter veränderten Orientierungsbedingungen. J. comp. Physiol. 99, 119-138.

HoEfer I., Lindauer M., 1976. - Der Einfluss einer Vordressur auf das Lernverhalten der Honigbiene. $J$. comp. Physiol. 109 (1), 249-264.

Hoefer I., Lindauer M., 1976. - Der Schatten als Hilfsmarke bei der Orientierung der Honigbiene. J. comp. Physiol. 112 (2), 5-18.

LAUer J., Lindauer M., 1973. - Die Beteiligung von Lernprozessen bei der Orientierung. Sonderdruck aus " Fortschritte der Zoologie " Band 21 Heft 2/3, Gustav Fischer Verlag, Stuttgart.

LINDAUER M., 1970. - Lernen und Gedächtnis - Versuche an der Honigbiene Die Naturwissenschaften 57 , 463-467. 
MENZEL R., 1967. - Untersuchungen zum Erlernen von Spektralfarben durch die Honigbiene (Apis mellifica). J. comp. Physiol. 56, 22-62.

Menzel R., 1968. - Das Gedächtnis der Honigbiene für Spektralfarben. J. comp. Phy'siol. 60, 82-102.

MeNzel R., ERber J., 1972. - The influence of the quantity of reward on the learning performance in honeybees. Behaviour 41, 27-42.

Menzel R., Freudel H., Rühl U., 1973. - Rassenspezifische Unterschiede im Lernverhalten der Honigbiene (Apis mellifica). Apidologie 4 (1), 1-24.

Menzel R., Erber J., MASuhr T., 1974. - Learning and memory in the honeybee. In : Experimental Analysis of Insect Behaviour. Ed. L. Barton-Brown Springer Verlag, Berlin-New York.

Plass F., 1953. - Inzuchtwirkung und Heterosiseffekt bei der Honigbiene. Schriftreihe der AID, H. 66 Bonn.

WAerden B. L. van der, Nievergelt E., 1956. - Tafeln zum Vergleich zweier Stichproben mittels x-Test und Zeichentest. Springer Verlag, Heidelberg-Berlin.

WoYKe J., 1965. - Genetic proof of the origin of drones from the fertilized eggs of the honeybee. J. Apic. Res. 4, 7-11. 